\title{
A STRONGLY STRICT NEGATIVE-IMAGINARY LEMMA FOR NON-MINIMAL LINEAR SYSTEMS*
}

\author{
ALEXANDER LANZON ${ }^{\ddagger}$, ZHUOYUE SONG ${ }^{\ddagger}$, SOURAV PATRA ${ }^{\ddagger}$, AND
}

IAN R. PETERSEN§

\begin{abstract}
A state-space characterization is given for strongly strict negative-imaginary systems. It facilitates both robust analysis and synthesis methods for interconnected negative-imaginary systems. Numerical advantages are achieved by avoiding a non-convex rank constraint, a non-strict inequality condition and a minimality assumption present in previous literature.
\end{abstract}

Key words: negative-imaginary systems, positive-real, non-minimal realization

Notation. Notation is standard. $\mathcal{R}$ and $\mathcal{R} \mathcal{H}_{\infty}$ denote the set of all proper realrational and proper real-rational stable transfer function matrices, respectively. $\mathbb{R}$ and $\mathbb{C}$ are the fields of real and complex numbers, respectively. The superscript $(\cdot)^{n \times m}$ denotes an operator with $m$ columns and $n$ rows. $\operatorname{Re}[s]$ and $\operatorname{Im}[s]$ denote the real and imaginary part of a complex number $s \in \mathbb{C}$, respectively. Furthermore, let $A^{*}$ be the complex conjugate transpose of matrix $A$, and let $\operatorname{det}(A)$ be the determinant of a square matrix $A$.

1. Introduction. Negative-imaginary (NI) systems appear in many engineering applications, for example, the large space structures, multi-link robots, DC machines, active filters etc ( $[6,7,12,13,16-18,21,22,26-28]$ and references therein). These systems are Lyapunov stable systems (with no poles at the origin) with an equal number of inputs and outputs satisfying the frequency domain condition: $j\left[R(j \omega)-R^{*}(j \omega)\right] \geq$ 0 for all $\omega \in(0, \infty)[12,13,17,28]$, where $R(s)$ is the transfer function matrix of the system. Strictly negative-imaginary (SNI) systems are square asymptotically stable systems that satisfy a strict negative-imaginary condition: $j\left[R(j \omega)-R^{*}(j \omega)\right]>0$ for all $\omega \in(0, \infty)[12,13]$. The positive frequency branch of the Nyquist plot of a typical NI system is shown in Fig. 1.1. This figure illustrates (in a SISO setting) that the imaginary part of the frequency response of the positive frequency branch is negative. All real-rational systems are real at zero frequency and at infinite frequency, so all NI systems must touch the real axis at zero and infinite frequency. NI systems can additionally touch the real axis at frequencies other than zero and infinite frequency.

\footnotetext{
* Dedicated to Brian Anderson on the occasion of his 70th birthday. The financial support of the Engineering and Physical Sciences Research Council and the Royal Society is gratefully acknowledged.

${ }^{\dagger}$ To whom correspondence should be addressed. E-mail: alexander.lanzon@manchester.ac.uk

${ }^{\ddagger}$ Control Systems Centre, School of Electrical and Electronic Engineering, University of Manchester, Manchester M13 9PL, UK.

§School of Information Technology and Electrical Engineering, University of New South Wales at the Australian Defence Force Academy, Canberra, ACT 2006 Australia.
} 


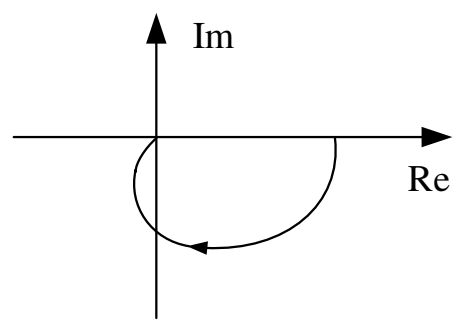

FIG. 1.1. An example of the Nyquist plot for NI systems.

For SNI systems, the Nyquist plot cannot touch the real axis except at zero frequency and infinity.

The concept of NI systems is similar to that of positive real (PR) systems where the frequency response is constrained to lie in one half of the complex plane $[1,3,4]$. In this regard, one might think that NI systems and PR systems are related by a simple rotational transformation, however, this is not always true. From associated frequency conditions, it is apparent that an NI system can be transformed into a PR system by multiplying the transfer function matrix with $-\frac{1}{s} I$ or $s I$ under some technical assumptions. However, the former transformation raises instability issues and difficulties in invoking computational results that rely on asymptotically stable systems, and the latter may cause for improperness of the transformed system but more importantly also introduces a blocking zero at zero frequency. This blocking zero via the latter transformation results into an SNI system being always transformed into a non-strict PR system as opposed to a strict PR system as one would expect or hope for. Hence, the passivity theorem $[11,14]$ cannot capture the stability of the interconnection of NI systems because after the transformation both of the systems are always transformed into two PR systems instead of a PR system and a strictly positivereal (SPR) system. Furthermore, approaches based on SPR synthesis (see $[8,23,25]$ ) cannot be used for control of an NI system irrespective of whether it is strict or non-strict NI due to the aforementioned difficulties.

A robust stability analysis result for interconnected NI systems was proposed in $[12,13,17,28]$. A positive interconnection of NI systems is internally stable if and only if the DC loop-gain is contractive provided that one of the systems is SNI and some technical assumptions hold $[12,13,17,28]$. This robust stability analysis result is a conditional stability result on the DC loop gain and is hence different from unconditional stability results such as the small-gain theorem [29] and the passivity theorem $[11,14]$.

In both analysis and synthesis frameworks related to NI systems, ensuring an SNI property is hence essential. For example, in a linear fractional transformation (LFT) framework [20-22], the interconnected system is internally stable in the presence of NI uncertainty provided that the rest of the closed-loop system is SNI and the DC loop 
gain is contractive. The original NI lemma (with its necessary and sufficient statespace characterization) was proposed in [13] and later extended in [28] to allow $j \omega$-axis poles except at the origin. The present work provides a state-space characterization for checking the SNI property of systems. An alternate characterization proposed in $[17,28]$ is referred to as the "Weakly Strict Negative-Imaginary (WSNI) Lemma" as it is derived via an underpinning weakly strict positive-real (WSPR) property of the system [15]. The WSNI lemma in [17,28] is difficult to apply for NI controller synthesis, for example, as it requires a minimality assumption and a non-convex rank condition to be fulfilled on a punctured $j \omega$-axis. However, in this work these difficulties have been circumvented. The proposed SNI lemma is referred to as the "Strongly Strict Negative-Imaginary (SSNI) Lemma" as it is developed via an underpinning strongly strict positive-real (SSPR) result [9].

The contributions of this work are as follows: the minimality assumption required in all previous versions of NI or WSNI lemmas is now relaxed, and hence a statespace characterization is given for SSNI systems. This relaxation facilitates controller synthesis as the minimality assumption cannot be computed 'a priori' in controller synthesis to satisfy the SNI property of the synthesized loop, which is necessary for robust stability of the closed-loop system. The proposed SSNI characterization also gives numerical advantages by avoiding the non-convex rank constraint and the nonstrict inequality which are present in previous literature $[13,17,28]$. The rest of the paper is structured as follows: Section 2 provides some background material that helps to streamline the main results of this paper. The Strongly Strict NegativeImaginary Lemma is presented in Section 3. In Section 4, a numerical example is given to illustrate the proposed results. Finally, Section 5 concludes the paper.

2. Preliminaries. First, we recall the definition of NI systems and SSPR systems as follows:

Definition 2.1. [28] A real-rational proper transfer function matrix $R(s) \in$ $\mathcal{R}^{m \times m}$ is said to be NI if

1) $R(\infty)=R^{T}(\infty)$

2) $R(s)$ has no poles at the origin and in $\mathbf{R e}[s]>0$;

3) $j\left[R(j \omega)-R(j \omega)^{*}\right] \geq 0$ for all $\omega \in(0, \infty)$ except the values of $\omega$ where $j \omega$ is a pole of $R(s)$.

4) If $j \omega_{0}$ is a pole of $R(s)$, it is at most a simple pole and the residue matrix $K_{0} \triangleq$ $\lim _{s \rightarrow j \omega_{0}}\left(s-j \omega_{0}\right) s(R(s)-R(\infty))$ is positive-semidefinite Hermitian.

DeFinition 2.2. [13,28] A real-rational proper transfer function matrix $R(s) \in$ $\mathcal{R}^{m \times m}$ is said to be SNI if

1) $R(\infty)=R^{T}(\infty)$

2) $R(s)$ has no poles in $\mathbf{R e}[s] \geq 0$;

3) $j\left[R(j \omega)-R(j \omega)^{*}\right]>0$ for all $\omega \in(0, \infty)$. 
DEFINITION 2.3. [5] A real-rational proper transfer function matrix $G(s) \in \mathcal{R}^{m \times m}$ is SSPR if

1) $G(s)$ has no poles in $\mathbf{R e}[s] \geq 0$,

2) $G(j \omega)+G(j \omega)^{*}>0$ for all $\omega \in \mathbb{R}$,

3) $\lim _{\omega \rightarrow \infty} \omega^{2 \rho} \operatorname{det}\left(G(j \omega)+G(j \omega)^{*}\right)>0$ where $\rho$ is the dimension of the null space of $G(\infty)+G(\infty)^{T}$.

REMARK 2.4. [5] For strictly proper transfer functions, condition 3) in Definition 2.3 reduces to $\lim _{\omega \rightarrow \infty} \omega^{2}\left(G(j \omega)+G(j \omega)^{*}\right)>0$ which coincides with the condition previously presented in the literature (see [2, 5, 9, 11, 24] for details).

Note that the frequency domain properties of NI systems are defined in the frequency interval $\omega \in(0, \infty)$, while for SSPR systems the frequency domain properties are fulfilled on the entire $j \omega$-axis.

The following lemma gives a state-space characterization for an SSPR property of a system. The standard Strictly Positive Real Lemma is given for minimal systems (for example in [4]), however, the following lemma is given for non-minimal systems. This lemma will be used in the next section to develop the main results of this paper.

Lemma 2.5. Let $G(s)=C(s I-A)^{-1} B$ be a strictly proper $m \times m$ transfer function matrix and $G(s)+G(-s)^{T}$ has normal rank $m$.

(i) If there exists a matrix $P=P^{T}>0$ that satisfies

$$
\begin{array}{r}
P A+A^{T} P<0, \\
P B=C^{T},
\end{array}
$$

then $A$ is Hurwitz and $G(s)$ is SSPR.

(ii) Suppose $(A, B)$ is controllable. If $A$ is Hurwitz and $G(s)=C(s I-A)^{-1} B$ is SSPR, then there exists a matrix $P=P^{T}>0$ that satisfies conditions in (2.1) and (2.2).

(iii) Suppose $(C, A)$ is observable. If $A$ is Hurwitz and $G(s)=C(s I-A)^{-1} B$ is SSPR, then there exists a matrix $P=P^{T}>0$ that satisfies conditions in (2.1) and (2.2).

(iv) Suppose the state-space realization $(A, B, C)$ has no observable uncontrollable modes. If $A$ is Hurwitz and $G(s)=C(s I-A)^{-1} B$ is SSPR, then there exists a matrix $P=P^{T}>0$ that satisfies conditions in (2.1) and (2.2).

Proof. (i) The proof is omitted as it is similar to the proof of the sufficiency part of Lemma 6.3 in [11].

(ii) This can be readily obtained via Theorem 3.1 of [24].

(iii) This can be readily obtained via Corollary 3.1 of [24].

(iv) Note that the state-space realization $(A, B, C)$ has no observable uncontrollable modes, hence, without loss of generality, we suppose the state-space realization 
$(A, B, C)$ is with the following Kalman canonical form:

$$
G:=\left[\begin{array}{c|c}
A & B \\
\hline C & 0
\end{array}\right]=\left[\begin{array}{ccc|c}
A_{11} & 0 & 0 & B_{1} \\
A_{21} & A_{22} & A_{23} & B_{2} \\
0 & 0 & A_{33} & 0 \\
\hline C_{1} & 0 & 0 & 0
\end{array}\right],
$$

where the eigenvalues of $A_{11}$ are controllable and observable modes, the eigenvalues of $A_{22}$ are controllable but unobservable modes, and the eigenvalues of $A_{33}$ are uncontrollable and unobservable modes of the sate-space realization $(A, B, C)$. Also, note that $G(s)$ is SSPR implies

$$
\begin{aligned}
\hat{G}(s):= & \hat{C}(s I-A)^{-1} \hat{B}+\hat{D} \\
& {\left[\begin{array}{ccc|ccc}
A_{11} & 0 & 0 & B_{1} & 0 & 0 \\
A_{21} & A_{22} & A_{23} & B_{2} & I & 0 \\
0 & 0 & A_{33} & 0 & 0 & I \\
\hline C_{1} & 0 & 0 & 0 & 0 & 0 \\
0 & 0 & 0 & 0 & I & 0 \\
0 & 0 & 0 & 0 & 0 & I
\end{array}\right] } \\
= & {\left[\begin{array}{ccc}
G(s) & 0 & 0 \\
0 & I & 0 \\
0 & 0 & I
\end{array}\right] }
\end{aligned}
$$

is SSPR. Also, note that $A$ is Hurwitz and $(A, \hat{B})$ is controllable, it follows from Theorem 3.1 of [24] that there exist $\hat{P}=\hat{P}^{T}>0$ and $L=L^{T}>0, \varepsilon>0$ and real matrices $Q$ and $W$ such that

$$
\begin{aligned}
\hat{P} A+A^{T} \hat{P} & =-Q Q^{T}-\varepsilon L, \\
\hat{C}-\hat{B}^{T} \hat{P} & =W^{T} Q \\
\hat{D}+\hat{D}^{T} & =W^{T} W .
\end{aligned}
$$

Partitioning $W$ as $W=\left[\begin{array}{lll}W_{1} & W_{2} & W_{3}\end{array}\right]$ with compatible dimension, we have $W_{1}=0$ as the $(1,1)$ block of $\hat{D}$ is zero. Considering the part of $(2.3)$ and $(2.4)$ corresponding to $(1,1)$ block of $\hat{G}(s)$, namely $G(s)$, we obtain that there exist $\hat{P}=$ $\hat{P}^{T}>0, L=L^{T}>0, \varepsilon>0$ and real matrix $Q$ such that

$$
\begin{aligned}
\hat{P} A+A^{T} \hat{P} & =-Q Q^{T}-\varepsilon L, \\
C-B^{T} \hat{P} & =0
\end{aligned}
$$

which implies that there exists $P=\hat{P}=P^{T}>0$ that satisfies conditions in (2.1) and $(2.2)$.

REMARK 2.6. The assumption that $G(s)+G(-s)^{T}$ has normal rank $m$ is in order to avoid redundances in inputs and/or outputs [11]. 
Now, a state-space realization of the reciprocal system will be given under the assumption that the state-space realization for the original system has no poles at the origin. This lemma will be invoked later to transform a system with a blocking zero at zero frequency into a strictly proper system.

Lemma 2.7. Suppose a square transfer function matrix $G(s) \in \mathcal{R}^{m \times m}$ has a state-space realization $(A, B, C, D)$ with $A \in \mathbb{R}^{n \times n}, B \in \mathbb{R}^{n \times m}, C \in \mathbb{R}^{m \times n}$ and $D \in \mathbb{R}^{m \times m}$. Suppose $\operatorname{det}(A) \neq 0$. Then $(\bar{A}, \bar{B}, \bar{C}, \bar{D})$ is a state-space realization of $G\left(\frac{1}{s}\right)$, where

$$
\bar{A}=A^{-1}, \bar{B}=-A^{-1} B, \bar{C}=C A^{-1}, \bar{D}=D-C A^{-1} B .
$$

Proof. From (2.6), we can obtain that

$$
\begin{aligned}
\bar{D}+\bar{C}(s I-\bar{A})^{-1} \bar{B} & =D-C A^{-1} B+C A^{-1}\left(s I-A^{-1}\right)^{-1}\left(-A^{-1} B\right) \\
& =D-C A^{-1}\left[I+\left(s I-A^{-1}\right)^{-1} A^{-1}\right] B \\
& =D-C A^{-1}(s A-I)^{-1} s A B \\
& =D+C\left(\frac{1}{s} I-A\right)^{-1} B .
\end{aligned}
$$

This implies that $(\bar{A}, \bar{B}, \bar{C}, \bar{D})$ is a state-space realization of $G\left(\frac{1}{s}\right)$ by noting that $(A, B, C, D)$ is a state-space realization of $G(s)$.

3. Main Result. A state-space characterization for the strongly strict negativeimaginary property of a system is given in this section. The main theorem is derived via the SSPR property of a transformed system; and before stating the main result, two technical lemmas are presented to streamline the proof of the main theorem.

Lemma 3.1. Given $R(s) \in \mathcal{R}^{m \times m}$ with $R(\infty)=R(\infty)^{T}$. The following statements are equivalent:

(1) $R(s)$ is $S N I$;

(2) $\hat{R}(s)=R(s)-R(\infty)$ is $S N I$;

(3) $G(s)=s(R(s)-R(\infty)) \in \mathcal{R H}_{\infty}, G(0)=0$ and $G(j \omega)+G(j \omega)^{*}>0 \forall \omega \in(0, \infty)$.

Proof. This trivially follows via Definition 2.2.

The above lemma states that an SNI system $R(s)$ can be transformed into an equivalent system $G(s)$ with a blocking zero at the origin that satisfies the strictly positive-real frequency condition $G(j \omega)+G(j \omega)^{*}>0$ in the frequency interval $\omega \in$ $(0, \infty)$. Because of this blocking zero condition, the existing SPR Lemmas (strong [2] [11], extended [23], marginally stable [10], weakly [15]) cannot provide any useful solution for state-space characterizations of the SNI systems.

To this end, the following lemma, however, can provide a solution via the SSPR property of the reciprocal system $G\left(\frac{1}{s}\right)$. The use of a reciprocal system is key to this work. Using this concept, the blocking zero condition of $G(s)$ at zero frequency has been transformed into the strictly proper condition of its reciprocal system. Also, 
$G\left(j \frac{1}{\omega}\right)+G\left(j \frac{1}{\omega}\right)^{*}>0 \forall \omega \in(0, \infty)$ is equivalent to $G(j \omega)+G(j \omega)^{*}>0 \forall \omega \in(0, \infty)[19]$. These results are presented in the following lemma:

Lemma 3.2. Let $(A, B, C, D)$ be the state-space realization of an $m \times m$ transfer function matrix $G(s)$ and $G(s)+G(-s)^{T}$ has normal rank $m$. Suppose $\operatorname{det}(A) \neq 0$.

(i) If $D-C A^{-1} B=0$ and there exists a matrix $Y=Y^{T}>0$ that satisfies

$$
\begin{array}{r}
A Y+Y A^{T}<0, \\
\text { and } B=-A Y A^{-T} C^{T},
\end{array}
$$

then $A$ is Hurwitz, and

$$
\begin{array}{r}
G(s) \in \mathcal{R H}_{\infty}, G(0)=0, G(j \omega)+G(j \omega)^{*}>0 \forall \omega \in(0, \infty), \\
G(\infty)+G(\infty)^{*}>0 \text { and } \lim _{\omega \rightarrow 0} \frac{1}{\omega^{2}}\left(G(j \omega)+G(j \omega)^{*}\right)>0 .
\end{array}
$$

(ii) Suppose $(C, A)$ is observable. If $A$ is Hurwitz and the conditions in (3.3) are satisfied, then $D-C A^{-1} B=0$ and there exists a matrix $Y=Y^{T}>0$ that satisfies (3.1) and (3.2).

(iii) Suppose $(A, B, C, D)$ has no observable uncontrollable modes. If $A$ is Hurwitz and the conditions in (3.3) are satisfied, then $D-C A^{-1} B=0$ and there exists a matrix $Y=Y^{T}>0$ that satisfies (3.1) and (3.2).

Proof. (i) Since there exists a matrix $Y=Y^{T}>0$ such that conditions in (3.1) and (3.2) are satisfied, it follows that there exists $P=Y^{-1}>0$ such that

$$
\begin{aligned}
& P A^{-1}+\left(A^{-1}\right)^{T} P<0, \\
& P\left(-A^{-1} B\right)=\left(C A^{-1}\right)^{T} .
\end{aligned}
$$

Also, since $D-C A^{-1} B=0$, it follows from Lemma $2.5(i)$ and Lemma 2.7 that the strictly proper transfer function $\tilde{G}(s)=G\left(\frac{1}{s}\right)$ is SSPR. Then, via Definition 2.3, we have $\tilde{G}(j \omega)+\tilde{G}(j \omega)^{*}>0 \forall \omega \in \mathbb{R}$ and $\lim _{\omega \rightarrow \infty} \omega^{2}\left(\tilde{G}(j \omega)+\tilde{G}(j \omega)^{*}\right)>0$ which implies $G\left(\frac{1}{j \omega}\right)+G\left(\frac{1}{j \omega}\right)^{*}>0 \forall \omega \in[0, \infty)$ and $\lim _{\omega \rightarrow \infty} \omega^{2}\left(G\left(\frac{1}{j \omega}\right)+G\left(\frac{1}{j \omega}\right)^{*}\right)>0$. Also, note that

$$
\begin{aligned}
& G\left(\frac{1}{j \omega}\right)+G\left(\frac{1}{j \omega}\right)^{*}>0 \forall \omega \in(0, \infty) \\
\Leftrightarrow & G\left(-j \frac{1}{\omega}\right)+G\left(-j \frac{1}{\omega}\right)^{*}>0 \forall \omega \in(0, \infty) \\
\Leftrightarrow & G\left(-j \frac{1}{\omega}\right)+G\left(j \frac{1}{\omega}\right)^{T}>0 \forall \omega \in(0, \infty) \\
\Leftrightarrow & G\left(j \frac{1}{\omega}\right)+G\left(-j \frac{1}{\omega}\right)^{T}>0 \forall \omega \in(0, \infty) \\
\Leftrightarrow & G(j \omega)+G(j \omega)^{*}>0 \forall \omega \in(0, \infty) .
\end{aligned}
$$

Similarly,

$$
\lim _{\omega \rightarrow 0}\left(G\left(\frac{1}{j \omega}\right)+G\left(\frac{1}{j \omega}\right)^{*}\right)=\left[G\left(\frac{1}{s}\right)+G\left(-\frac{1}{s}\right)^{T}\right](0)>0 \Leftrightarrow G(\infty)+G(\infty)^{*}>0,
$$




$$
\lim _{\omega \rightarrow \infty} \omega^{2}\left(G\left(\frac{1}{j \omega}\right)+G\left(\frac{1}{j \omega}\right)^{*}\right)>0 \Leftrightarrow \lim _{\omega \rightarrow 0} \frac{1}{\omega^{2}}\left(G(j \omega)+G(j \omega)^{*}\right)>0 .
$$

Hence, $G(j \omega)+G(j \omega)^{*}>0 \forall \omega \in(0, \infty), G(\infty)+G(\infty)^{*}>0$ and $\lim _{\omega \rightarrow 0} \frac{1}{\omega^{2}}(G(j \omega)+$ $\left.G(j \omega)^{*}\right)>0$. Also, $D-C A^{-1} B=0$ implies $G(0)=0$. Finally, condition (3.1) implies that $A$ is Hurwitz and $G(s) \in \mathcal{R H}_{\infty}$.

(ii) First note that the conditions in (3.3) are satisfied, it follows from (3.6), (3.7) and (3.8) that $G\left(\frac{1}{j \omega}\right)+G\left(\frac{1}{j \omega}\right)^{*}>0 \forall \omega \in[0, \infty)$ and $\lim _{\omega \rightarrow \infty} \omega^{2}\left(G\left(\frac{1}{j \omega}\right)+G\left(\frac{1}{j \omega}\right)^{*}\right)>0$. Also, since $G(0)=0$, it follows from Lemma 2.7 that $\tilde{G}(s)=G\left(\frac{1}{s}\right)$ satisfies $\tilde{G}(\infty)=$ $D-C A^{-1} B=G(0)=0$. Furthermore, since $A$ is Hurwitz, it follows that $A^{-1}$ is Hurwitz which implies $G\left(\frac{1}{s}\right) \in \mathcal{R} \mathcal{H}_{\infty}$ via Lemma 2.7. Consequently, $G\left(\frac{1}{s}\right)$ is SSPR via Definition 2.3. Using Lemma 2.7, $(\bar{A}, \bar{B}, \bar{C}, \bar{D})$ is a state-space realization of $G\left(\frac{1}{s}\right)$, where $(\bar{A}, \bar{B}, \bar{C}, \bar{D})$ is given in (2.6). Also, note that $(C, A)$ being observable implies that $(\bar{C}, \bar{A})$ is observable by noting that $A$ is nonsingular. Then, it follows from the SSPR Lemma (Lemma 2.5 (iii)) that there exists a matrix $P=P^{T}>0$ that satisfies conditions in (3.4) and (3.5). Let $Y=P^{-1}$, then the condition in (3.5) is equivalent to (3.2). Finally, it follows via a simple algebraic computation that condition (3.4) is equivalent to (3.1).

(iii) First note $A$ is nonsingular as $A$ is Hurwitz. Also, note that the statement that $(A, B, C, D)$ has no observable uncontrollable modes implies that $(\bar{A}, \bar{B}, \bar{C}, \bar{D})$ has no observable uncontrollable modes when $A$ is nonsingular, where $(\bar{A}, \bar{B}, \bar{C}, \bar{D})$ is given in (2.6). Then, it follows the same lines of the proof of (ii) with only replacement of invoking Lemma 2.5 (iv) instead of Lemma 2.5 (iii).

As a consequence of Lemma 3.1 and Lemma 3.2, we obtain the following main theorem characterizing properties of strictly negative-imaginary systems. In contrast to the Weakly Strict Negative-Imaginary Lemma [28], we refer to this theorem as the Strongly Strict Negative-Imaginary Lemma.

THEOREM 3.3. (SSNI Lemma) Given a square transfer function matrix $R(s) \in$ $\mathcal{R}^{m \times m}$ with a state-space realization $(A, B, C, D)$, where $A \in \mathbb{R}^{n \times n}, B \in \mathbb{R}^{n \times m}$, $C \in \mathbb{R}^{m \times n}$ and $D \in \mathbb{R}^{m \times m}$. Suppose $R(s)+R(-s)^{T}$ has normal rank $m$ and $(C, A)$ is observable. Then, $A$ is Hurwitz and $R(s)$ is SNI with

$$
\lim _{\omega \rightarrow \infty} j \omega\left(R(j \omega)-R(j \omega)^{*}\right)>0 \text { and } \lim _{\omega \rightarrow 0} j \frac{1}{\omega}\left(R(j \omega)-R(j \omega)^{*}\right)>0
$$

if and only if $D=D^{T}$ and there exists a matrix $Y=Y^{T}>0$ such that

$$
A Y+Y A^{T}<0 \text { and } B=-A Y C^{T} .
$$

Proof. $(\Rightarrow)$ Since $R(s)$ is SNI, we have $D=D^{T}$ via Lemma 1 of [13]. Then, it follows from Lemma 3.1 that $G(s)=s(R(s)-R(\infty))$ satisfying $G(s) \in \mathcal{R H}_{\infty}$, 
$G(0)=0$ and $G(j \omega)+G(j \omega)^{*}>0 \forall \omega \in(0, \infty)$. Also note

$$
\begin{aligned}
& \lim _{\omega \rightarrow \infty} j \omega\left(R(j \omega)-R(j \omega)^{*}\right)>0 \\
\Leftrightarrow & \lim _{\omega \rightarrow \infty}\left[j \omega(R(j \omega)-R(\infty))+(j \omega(R(j \omega)-R(\infty)))^{*}\right]>0 \\
& \left(\operatorname{noting} R(\infty)=R(\infty)^{T}\right) \\
\Leftrightarrow & \lim _{\omega \rightarrow \infty} G(j \omega)+G(j \omega)^{*}>0 \\
\Leftrightarrow & G(\infty)+G(\infty)^{T}>0 .
\end{aligned}
$$

Similarly,

$$
\lim _{\omega \rightarrow 0} j \frac{1}{\omega}\left(R(j \omega)-R(j \omega)^{*}\right)>0 \Leftrightarrow \lim _{\omega \rightarrow 0} \frac{1}{\omega^{2}}\left(G(j \omega)+G(j \omega)^{*}\right)>0 .
$$

Hence $G(s)=s(R(s)-R(\infty))$ satisfies the conditions in (3.3). Also, since $(A, B, C A, C B)$ is a state-space realization for $G(s)$, and $(C A, A)$ is observable by noting $(C, A)$ is observable and $A$ is Hurwitz, then via Lemma 3.2 (ii), condition (3.3) implies that there exists a matrix $Y=Y^{T}>0$ such that

$$
A Y+Y A^{T}<0 \text { and } B=-A Y A^{-T}(C A)^{T}=-A Y C^{T} .
$$

$(\Leftarrow)$ Since there exists a matrix $Y=Y^{T}>0$ that satisfies conditions in (3.10), it follows that $A$ is Hurwitz, which implies that $A$ is nonsingular. Hence, (3.10) can be rewritten as $A Y+Y A^{T}<0$ and $B=-A Y A^{-T}(C A)^{T}$. Also, since $(A, B, C A, C B)$ is a state-space realization for $G(s)=s(R(s)-R(\infty))$ and note that $C B-C A(A)^{-1} B=$ 0 , via Lemma $3.2(i)$, it follows that $G(s)=s(R(s)-R(\infty))$ satisfies the conditions in (3.3). Also note $D=D^{T}$, hence, it follows from Lemma 3.1, (3.11) and (3.12) that $R(s)$ satisfies SNI property and conditions in (3.9), which completes the proof.

TheOREM 3.4. (SSNI Lemma) Given a square transfer function matrix $R(s) \in$ $\mathcal{R}^{m \times m}$ with a state-space realization $(A, B, C, D)$, where $A \in \mathbb{R}^{n \times n}, B \in \mathbb{R}^{n \times m}, C \in$ $\mathbb{R}^{m \times n}$ and $D \in \mathbb{R}^{m \times m}$. Suppose $R(s)+R(-s)^{T}$ has normal rank $m$ and $(A, B, C, D)$ has no observable uncontrollable modes. Then, $A$ is Hurwitz and $R(s)$ is SNI with (3.9) satisfied if and only if $D=D^{T}$ and there exists a matrix $Y=Y^{T}>0$ that satisfies conditions in (3.10).

Proof. First note that the statement that $(A, B, C, D)$ has no observable uncontrollable modes implies that the state-space realization of $G(s)=s(R(s)-R(\infty))$ : ( $A, B, C A, C B)$ has no observable uncontrollable modes when $A$ is nonsingular. Then, the results follow from the same proof lines of Theorem 3.3 with only replacement of invoking Lemma $3.2(i)$ and Lemma 3.2 (iii) instead of Lemma $3.2(i)$ and Lemma $3.2($ ii) .

REMARK 3.5. The assumption that $(C, A)$ is observable in Theorem 3.3 is only needed to prove necessity part of the theorem. Alternatively, the assumption that $(A, B, C, D)$ has no observable uncontrollable modes is another necessary requirement to show the SNI property as posed in Theorem 3.4. 
REMARK 3.6. Theorem 3.3 and Theorem 3.4 can also be proven via the alternate transformation, $\bar{G}(s)=-\frac{1}{s}(R(s)-R(0))$ which relates the negative-imaginary property of a system with the positive-real property. Here, a sketch of the alternative proof is given as this may be instructive in its own right: First note that $\left(A, A^{-1} B,-C, 0\right)$ is a state-space realization for $\bar{G}(s)$, where $(A, B, C, D)$ is the state-space realization for $R(s)$. Also, note that the controllability and observability of $\left(A, A^{-1} B,-C\right)$ is the same as that of $(A, B, C)$. Then, it follows that the fulfillment of the conditions in (3.10) is equivalently implying the strictly proper system $\bar{G}(s)$ to be an SSPR system via Lemma 2.5. Then, via the definitions of SNI and SSPR systems, it can be shown that $\bar{G}(s)$ being an SSPR system is equivalent to $R(s)$ satisfying the SNI property and the conditions in (3.9), which completes the sketch of this proof.

Theorem 3.3 and Theorem 3.4 will enable robust control synthesis for uncertain NI systems. Via this result, an SNI controller can be synthesized by considering the simple algebraic conditions shown in (3.10) to stabilize an NI plant interconnected via positive feedback in a closed-loop $[13,17,28]$; or we can design a controller such that an LFT closed-loop system satisfies (3.10) to ensure the SNI property that facilitates robust stability against NI uncertainties. For robust stability, the DC loop gain should be contractive [13]. Note that existing results on robust control for uncertain NI systems typically only enforce a (non-strict) NI property on the closed-loop system comprising of the nominal plant and controller and thereby can only handle SNI uncertainty $[17,18,22]$, however, the uncertainties do not always satisfy the SNI property, see e.g. the example in [13]. This SSNI lemma will facilitate robust synthesis methods for NI systems and solve many numerical issues.

Next, we give some physical interpretations of the mathematical conditions in (3.9).

Lemma 3.7. Given $R(s)$ is a proper scalar SNI transfer function with $R(\infty) \geq 0$, then

$$
\lim _{\omega \rightarrow 0} j \frac{1}{\omega}\left(R(j \omega)-R(j \omega)^{*}\right)>0 \Leftrightarrow \lim _{\omega \rightarrow 0} \frac{d \phi(\omega)}{d \omega}<0,
$$

where $\phi(\omega)$ denote the phase of $R(j \omega)$.

Proof.

$$
\begin{aligned}
& \lim _{\omega \rightarrow 0} j \frac{1}{\omega}\left(R(j \omega)-R(j \omega)^{*}\right)>0 \\
\Leftrightarrow & \lim _{\omega \rightarrow 0} j \frac{1}{\omega}\left[r(\omega) e^{j \phi(w)}-r(\omega) e^{-j \phi(w)}\right]>0 \\
& \text { where } r(\omega) \text { denotes the magnitude of } R(j \omega)
\end{aligned}
$$




$$
\begin{aligned}
\Leftrightarrow & \lim _{\omega \rightarrow 0} j \frac{1}{\omega}(2 j \sin \phi(\omega))>0 \\
& \operatorname{noting} r(\omega)>0 \text { near } \omega=0 \text { since } R(0)>R(\infty) \geq 0[13] \\
\Leftrightarrow & \lim _{\omega \rightarrow 0} \frac{\sin \phi(\omega)}{\omega}<0 \\
\Leftrightarrow & \lim _{\omega \rightarrow 0} \cos \phi(\omega) \frac{d \phi(\omega)}{d \omega}<0 \\
& \text { since } R(0)>R(\infty) \geq 0[13], \text { hence } \phi(0)=0, \text { we can use L'Hospital's rule } \\
\Leftrightarrow & \lim _{\omega \rightarrow 0} \frac{d \phi(\omega)}{d \omega}<0 \text { noting } \cos \phi(\omega)>0 \text { in the neighborhood of } \omega=0 .
\end{aligned}
$$

The above lemma states that for proper scalar transfer function $R(s)$ with SNI property and $R(\infty) \geq 0, \lim _{\omega \rightarrow 0} j \frac{1}{\omega}\left(R(j \omega)-R(j \omega)^{*}\right)>0$ means that the phase of $R(j \omega)$ strictly decreases as frequency increases from $\omega=0$.

REMARK 3.8. For strictly proper scalar transfer functions, $\lim _{\omega \rightarrow \infty} j \omega(R(j \omega)-$ $\left.R(j \omega)^{*}\right)>0$ implies that $\operatorname{Im}[R(j \omega)]$ cannot go to zero faster than $\omega^{-1}$ when $|\omega| \rightarrow \infty$. This implies that the relative degree of $R(j \omega)$ must be zero or one.

As mentioned in Remark 3.8, if one uses the conditions in (3.10) to design an SNI controller, systems with relative degree two cannot be captured. Earlier (non-strict) NI lemmas $[13,17,28]$ invoke a non-strict Lyapunov inequality in (3.10) and yield a complete state-space characterization of (non-strict) NI systems. When the Lyapunov inequality in (3.10) becomes strict as in Theorem 3.3 (Theorem 3.4), then we get a complete state-space characterization of SNI systems but we also enforce a departure condition from and an arrival condition to the real axis as described by the limiting condition in (3.9). For example, $\frac{1}{s^{2}+2 s+2}$ and $\frac{2 s+2}{s^{2}+2 s+2}$ are two SNI systems, however, they violate the first and the second condition of (3.9), respectively.

4. Example. A numerical example is given to illustrate the main result of this paper. We consider a system $R(s)$ with a state-space realization

$$
\begin{aligned}
& {\left[\begin{array}{l}
\dot{x}_{1} \\
\dot{x}_{2} \\
\dot{x}_{3} \\
\dot{x}_{4}
\end{array}\right]=\left[\begin{array}{rrrr}
0 & 1 & 0 & 0 \\
0 & 0 & 1 & 0 \\
-5 & -9 & -5 & 0 \\
0 & 0 & 0 & -5
\end{array}\right]\left[\begin{array}{l}
x_{1} \\
x_{2} \\
x_{3} \\
x_{4}
\end{array}\right]+\left[\begin{array}{l}
0 \\
0 \\
1 \\
0
\end{array}\right] u, } \\
& y=\left[\begin{array}{llll}
9 & 6 & 1 & 1
\end{array}\right]\left[\begin{array}{l}
x_{1} \\
x_{2} \\
x_{3} \\
x_{4}
\end{array}\right]
\end{aligned}
$$

Note that this system has an uncontrollable mode $\{-5\}$, hence the results posed in $[13,17,28]$ cannot be applied to analyze the (strict) negative-imaginariness of this system with the given state-space realization. Since $R(s)+R(-s)^{T}$ has normal rank 


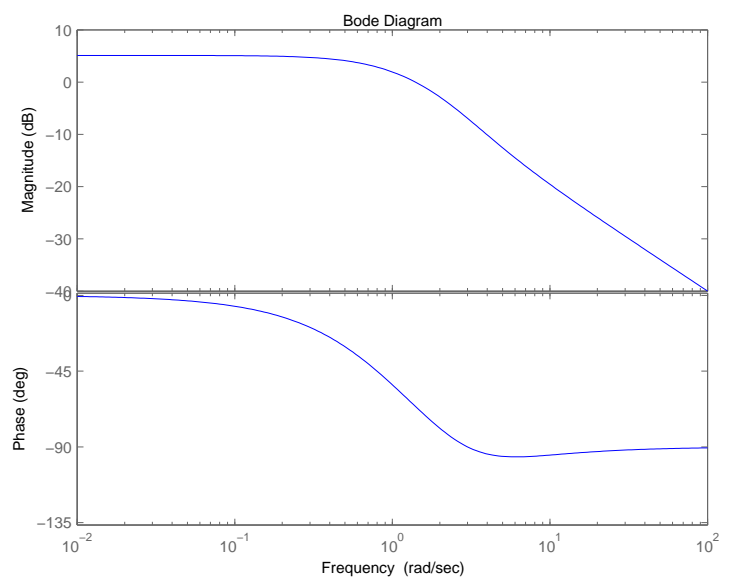

FIG. 4.1. Bode plot of the system

1, $(C, A)$ is observable and $D=0=D^{T}$ that satisfy the assumptions in Theorem 3.3, we can use Theorem 3.3 to analyze the NI property of the system.

YALMIP and SeDuMi are used to solve the conditions in (3.10) and we obtain the following solution

$$
Y=\left[\begin{array}{rrrr}
0.0958 & -0.1206 & 0.0908 & -0.0295 \\
-0.1206 & 0.2112 & -0.2081 & 0.0265 \\
0.0908 & -0.2081 & 0.4396 & -0.0082 \\
-0.0295 & 0.0265 & -0.0082 & 0.1146
\end{array}\right]>0
$$

Consequently, via Theorem 3.3, the system in (4.1) is SNI and also satisfies the conditions in (3.9).

The Bode plot of the transfer function $R(s)=\frac{s^{2}+6 s+9}{s^{3}+5 s^{2}+9 s+5}$ given in (4.1) is shown in Fig. 4.1. It is apparent that $R(s)$ is an SNI system since the phase $\phi \in(-\pi, 0)$ when the frequency varies from zero to infinity. Also, $\lim _{\omega \rightarrow \infty} j \omega\left(R(j \omega)-R(j \omega)^{*}\right)=2>0$ and $\lim _{\omega \rightarrow 0} j \frac{1}{\omega}\left(R(j \omega)-R(j \omega)^{*}\right)=4.08>0$ are satisfied, which coincides with the statement of the main theorem.

5. Conclusions. A state-space characterization for strongly strict negativeimaginary systems has been proposed. The results are derived using the strongly strict positive-real property of a transformed system. The proposed characterization relaxes the minimality assumption, which is different from [13,17,28]; and this relaxation facilitates analysis and controller synthesis methods for uncertain NI systems. Using this result, the robust analysis and synthesis frameworks can be extended for both the NI and SNI uncertainty of the system. This work also clarifies the relationship between the strict Lyapunov inequality (see (3.10)) and the SNI property of the system. The appealing prospect of linking NI/SNI/SSNI systems to circuit realization ideas will 
be investigated in future scope of the work using the well established connection to SPR/PR systems [4].

\section{REFERENCES}

[1] B. D. O. Anderson, A system theory criterion for positive real matrices, SIAM Journal on Control, 5 (1967), pp. 171-182.

[2] B. D. O. Anderson, M. Mansour, and F. J. Kraus., A new test for strict positive realness, IEEE Transactions on Circuits and Systems Part I, 42 (1995), pp. 226-229.

[3] B. D. O. Anderson and J. B. Moore, Algebraic structure of generalized positive real matrices, SIAM Journal on Control, 6 (1968), pp. 615-624.

[4] B. D. O. Anderson And S. Vongpanitlerd, Network Analysis and Synthesis: A Modern Systems Theory Approach, Prentice-Hall, Inc., Englewood Cliffs, NJ, 1973.

[5] M. Corless AND R. Shorten, On the characterization of strict positive realness for general matrix transfer functions, IEEE Transactions on Automatic Control, 55 (2010), pp. 18991904.

[6] S. Engelken, A. Lanzon, And I. R. Petersen, $\mu$ analysis for interconnections of systems with negative imaginary frequency response, in Proceedings of the 2009 European Control Conference, Budapest, Hungary, August 2009, pp. 555-560.

[7] S. Engelken, S. Patra, A. Lanzon, and I. R. Petersen, Stability analysis of negative imaginary systems with real parametric uncertainty - the SISO case, IET Control Theory and Applications, 4 (2010), pp. 2631-2638.

[8] J. C. Geromel and P. B. Gapski, Synthesis of positive real $\mathcal{H}_{2}$ controllers, IEEE Transactions on Automatic Control, 42 (1997), pp. 988-992.

[9] C. H. Huang, P. A. Ionnnou, J. Maroulas, and M. G. Safonov, Design of strictly positive real systems using constant output feedback, IEEE Transactions on Automatic Control, 44 (1999), pp. 569-573.

[10] S. M. Joshi And S. Gupta, On a class of marginally stable positive-real systems, IEEE Transactions on Automatic Control, 41 (1996), pp. 152-155.

[11] H. KhaliL, Nonlinear Systems, Prentice-Hall, Inc, 2nd ed., 1996.

[12] A. Lanzon and I. R. Petersen, A modified positive-real type stability condition, in Proceedings of the 2007 European Control Conference, Kos, Greece, July 2007, pp. 3912-3918.

[13] _ Stability robustness of a feedback interconnection of systems with negative imaginary frequency response, IEEE Transactions on Automatic Control, 53 (2008), pp. 1042-1046.

[14] R. Lozano, B. Brogliato, O. Egeland, and B. Maschke, Dissipative Systems Analysis and Control: Theory and Applications, Springer-Verlag New York, Inc., Secaucus, NJ, 2000.

[15] R. Lozano-Leal and S. M. Joshi, Strictly positive real transfer functions revisited, IEEE Transactions on Automatic Control, 35 (1990), pp. 1243-1245.

[16] S. Patra AND A. LANzon, Stability analysis of interconnected systems with mixed negativeimaginary and small-gain properties, IEEE Transactions on Automatic Control, in press.

[17] I. R. Petersen and A. Lanzon, Feedback control of negative-imaginary systems: Flexible structures with colocated actuators and sensors, IEEE Control Systems Magazine, 30 (2010), pp. 54-72.

[18] I. R. Petersen, A. Lanzon, And Z. Song, Stabilization of uncertain negative-imaginary systems via state-feedback control, in Proceedings of the 2009 European Control Conference, Budapest, Hungary, August 2009, pp. 1605-1609.

[19] R. Shorten, P. Curran, K. Wulff, and E. Zeheb, A note on spectral conditions for positive realness of transfer function matrices, IEEE Transactions on Automatic Control, 53 (2008), pp. $1258-1261$. 
[20] Z. Song, A. Lanzon, S. Patra, and I. R. Petersen, Robust performance analysis for uncertain negative-imaginary systems, International Journal of Robust and Nonlinear Control, in press.

[21] - Analysis of robust performance for uncertain negative-imaginary systems using structured singular value, in Proceedings of the 18th Mediterranean Conference on Control and Automation, Marrakech, Morocco, June 2010, pp. 1025-1030.

[22] — - Towards controller synthesis for systems with negative imaginary frequency response, IEEE Transactions on Automatic Control, 55 (2010), pp. 1506-1511.

[23] W. Sun, P. P. Khargonekar, and D. Shim, Solution to the positive real control problem for linear time-invariant systems, IEEE Transactions on Automatic Control, 39 (1994), pp. 2034-2046.

[24] G. TAO And P. A. Ionnnou, Strictly positive real matrices and the Lefschetz-KalmanYakubovich lemma, IEEE Transactions on Automatic Control, 33 (1988), pp. 1183-1185.

[25] L. Turan, M. G. Safonov, and C. H. Huang, Synthesis of positive real feedback systems: A simple derivation via Parrott's theorem, IEEE Transactions on Automatic Control, 42 (1997), pp. 1154-1157.

[26] J. Xiong, I. R. Petersen, and A. Lanzon, On lossless negative imaginary systems, in Proceedings of the 7th Asian Control Conference, Hong Kong, China, August 2009, pp. 824829.

[27] - Finite frequency negative imaginary systems, in Proceedings of the 2010 American Control Conference, Baltimore, MD, USA, Jun/Jul 2010, pp. 323-328.

[28] — A negative imaginary lemma and the stability of interconnections of linear negative imaginary systems, IEEE Transactions on Automatic Control, 55 (2010), pp. 2342-2347.

[29] K. Zhou, J. C. Doyle, and K. Glover, Robust and Optimal Control, Prentice-Hall, Inc., Saddle River, NJ, 1996. 